\title{
A roadmap for the future
}

\author{
The particle physics community refreshes the roadmap for the field in Europe, taking into account the worldwide \\ context, in the so-called European Strategy for Particle Physics update, which happens every seven years.
}

\author{
Fabiola Gianotti and Gian Francesco Giudice
}

W hat are the scientific priorities and future objectives of particle physics? Which instruments offer the best potential to achieve these objectives? And which technologies need to be developed to build such instruments? These are the main questions addressed by the European Strategy for Particle Physics (ESPP) following a bottom-up approach, driven by the scientific community.

The process is based on the physics knowledge at the time of the update, the results from existing facilities such as the Large Hadron Collider (LHC) at CERN, the status of the relevant technologies and the prospects for future advances, proposals and design studies for future experiments and facilities, and ideas for promising new avenues of investigation.

This input is scrutinised by the European Strategy Group, which includes representatives of all CERN's Member States and the directors of the major particle physics laboratories in Europe. The ESPP provides the scientific vision and priorities of the field for the short, medium and long terms, while also covering subjects of wider interest, such as education and public outreach, career prospects for young scientists, technology transfer, and the societal and environmental impact of particle physics. The Strategy update is a thorough and rigorous process, engaging the physics community for almost two years from beginning to end.

In June 2020, CERN's governing body, the Council, unanimously adopted the resolution to update the European Strategy, which provides a realistic and prudent approach to ambitious, visionary scientific objectives.

\section{The Higgs boson as a starting point \\ Recent experimental results, in particular from the LHC, have radically transformed the status of particle physics and form the basis for future research directions. The discovery of the Higgs boson has been a turning point, unveiling a particle with unprecedented characteristics and shedding new light on a phenomenon that has surprising similarities with}

the way certain materials behave as superconductors below a critical temperature.

It was fascinating to realize that the same phenomenon operates at cosmic scales, and made the early Universe undergo a phase transition that transformed the nature of empty space. Nevertheless, believing that the Higgs boson discovery has completed our understanding of this complex phenomenon is too simplistic. On the contrary, much remains to be understood about this very special particle, including whether it is an elementary or composite object, how it leads to the peculiar pattern of quark and lepton masses observed, what determines the stability of the vacuum and what triggered the phase transition in the early Universe.

These questions are still largely unexplored experimentally and raise deep conceptual concerns theoretically. That is why the ESPP update has identified the detailed study of the Higgs boson as the most pressing priority for the field. Since the Higgs boson discovery in 2012 the general-purpose LHC experiments, ATLAS and CMS, have made extraordinary progress in pinning down the features of this particle and, by the end of LHC operation in 2038 - thanks to the high-luminosity upgrades of the collider and the detectors - they should be able to measure the Higgs boson properties with greatly improved precision.

To gain even deeper insights into the Higgs boson and its role in fundamental physics, the ESPP recommends an electronpositron collider as the next facility, followed by a high-energy proton-proton collider in the longer term.

\section{A strategy for the future}

Humanity's thirst for knowledge, curiosity and spirit of exploration have always been the engines that drive particle physics. Unsurprisingly, the more we dive into uncharted territory the more difficult it becomes to predict what future experimental endeavours could find. This is the very essence of research: if we knew for certain what future experiments will discover, we would not need to build them.
The value of exploring the unknown does not lie in the number of hypothetical discoveries that are promised, but in the knowledge acquired through investigation and the guidance obtained on future research directions. From more than a century of research we have learned that nature hides its fundamental laws at the smallest scales - and that is what drives us to explore the innermost structure of matter.

Hence, for the longer term, the 2020 update of the ESPP identifies a protonproton collider reaching the highest energies allowed by technology (at least $100 \mathrm{TeV}$ ) as the most powerful tool for the exploration of uncharted territories. Even if it is difficult to anticipate what these studies will reveal, we can be certain that this path of exploration will greatly expand our knowledge on many fronts.

A good example of a guaranteed result is dark matter. A proton collider operating at energies around $100 \mathrm{TeV}$ will conclusively probe the existence of weakly interacting dark-matter particles of thermal origin. This will lead either to a sensational discovery or to an experimental exclusion that will profoundly influence both particle physics and astrophysics. Another example is the precision measurement of the Higgs boson self-interaction which, by confirming or refuting the underlying theory - the Standard Model - will significantly advance our understanding of the phase transition that occurred in the early Universe.

\section{Executing the strategy}

Given the size, complexity and cost of such a project, the ESPP recommends first to perform an assessment of its technical and financial feasibility. If this is successful, approval may be granted by the end of this decade. Construction would most likely take place in stages, and the cost of around $\$ 30$ billion, including the construction of a $100 \mathrm{~km}$ tunnel, the protonproton collider and an electron-positron Higgs and electroweak factory as the possible first stage, would be spread over 50 years or more.

Such an ambitious project can only be implemented through the collaboration of scientists from all over the world. It will 
require the development of cutting-edge technologies in several domains, from new-generation superconducting magnets to cryogenics, vacuum, electronics and the handling of big data with significant technological spin-offs for society.

Over the past 70 years, accelerators have contributed immensely to shaping our understanding of fundamental physics and the structure and evolution of the Universe. This came about not only through fascinating discoveries, but also through precise measurements of known particles, forces and phenomena. Precision measurements are not only a way of testing and consolidating known theories, but also an extremely powerful tool for detecting hints of new phenomena in a way that is complementary to and in some cases - more far-reaching than direct exploration.

A remarkable example is provided by the Large Electron-Positron collider at CERN and the Stanford Linear Collider at SLAC, which transformed our understanding of the Standard Model without discovering any new particles. The 2020 update of the ESPP reaffirms the crucial importance of precision measurements as discovery tools. This motivates a vast experimental programme not only at high-energy colliders, but also through a variety of lower-energy projects capable of probing known and new phenomena, for example, rare-decay or transition processes.

The ESPP also recommends support for accelerator-based neutrino projects in the US and Japan, theoretical research, and searches for very light, feebly interacting particles.

Another priority in the 2020 update of the ESPP is a reinforced accelerator research and development programme, with great potential for future applications in particle physics and other fields, focusing on new-generation superconducting high-field magnets and a variety of other technologies such as high-gradient accelerating structures, plasma wakefield acceleration, bright muon beams or energy-recovery linear accelerators. The ESPP also calls for intensified research and development on instrumentation, detectors, computing and software.
Continued, reinforced collaboration between European laboratories with partners in other regions of the world and with neighbouring fields such as nuclear and astroparticle physics is emphasised in the Strategy update as an indispensable tool for tackling extremely complex scientific questions. Last but not least, environmental protection is identified as a top priority with the recommendation that the impact of all future projects on the environment be carefully assessed and minimised, and that efforts be redoubled to save and re-use energy.

\section{Outlook}

Today, humanity is facing pressing challenges including climate change, combatting epidemics and poverty, confronting food and water shortages in many parts of the world, ensuring sustainable and clean energy production, and protecting the environment. Science is key to addressing these and other challenges and should be supported in all its facets. Investments in science are comparatively small on the macro-economic level, but their impact on the future of humanity is vast. In particular, history shows that fundamental research is a driver of innovation, and accelerator-based particle physics has spawned numerous technological advances that have benefitted society, from the World Wide Web to technologies for medical imaging and cancer treatment. Research at future colliders will boost technological developments in many fields in ways that are unimaginable without the driving force of fundamental science.

Our understanding of particle physics and cosmology has today reached an unprecedented level of maturity, and this has dramatically changed the targets of research. Although we have equations that describe most observed phenomena, we lack a true understanding of the principles underlying those equations and the physical origin of their many free parameters. Moreover, many of the open questions in the micro-world and the cosmos appear to be closely intertwined. For instance, some of the puzzles encountered in describing the current accelerating expansion of the Universe, and that which presumably took place in its early stages, show intriguing similarities with unexplained aspects related to the Higgs boson.

The focus of particle physics has thus evolved towards addressing structural questions about spacetime, fundamental interactions and the origin of the Universe. Some of these are as old as civilization itself and it is fascinating that we have today reached the maturity and developed the technologies to address them. The urge to seek answers to such questions is part of what defines us as humans. The ambitious task that lies ahead entails global collaboration on a courageous experimental venture, involving high-energy colliders, low-energy precision tests, observational cosmology, cosmic rays, dark-matter searches, gravitational waves, terrestrial and cosmic neutrinos, and much more.

Many different fields will enrich one another by sharing insights and experimental techniques, as the boundaries that used to separate them become increasingly blurred. In this global context, high-energy colliders will continue to be an indispensable and irreplaceable microscope to scrutinise nature at the smallest scales, providing knowledge that cannot be obtained through any other means.

Fabiola Gianotti ${ }^{\bowtie}$ and

Gian Francesco Giudice ${ }^{凶}$

CERN, Geneva, Switzerland.

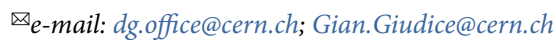

Published online: 24 September 2020

https://doi.org/10.1038/s41567-020-01054-6

Competing interests

The authors declare no competing interests.

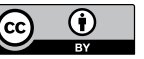

Open Access This article is licensed under a Creative Commons Attribution 4.0 International License, which permits use, sharing, adaptation, distribution and reproduction in any medium or format, as long as you give appropriate credit to the original author(s) and the source, provide a link to the Creative Commons license, and indicate if changes were made. The images or other third party material in this article are included in the article's Creative Commons license, unless indicated otherwise in a credit line to the material. If material is not included in the article's Creative Commons license and your intended use is not permitted by statutory regulation or exceeds the permitted use, you will need to obtain permission directly from the copyright holder. To view a copy of this license, visit http://creativecommons.org/licenses/by/4.0/. 